\title{
Investigating the concept of diffusional independence. Potential step transients at nano- and micro-electrode arrays: theory and experiment
}

\author{
Denis Menshykau, ${ }^{a}$ Xing-Jiu Huang, ${ }^{a}$ Neil V. Rees, ${ }^{a}$ F. Javier del Campo, ${ }^{b}$ Francesc Xavier Muñoz ${ }^{b}$ \\ and Richard G. Compton $* a$
}

\author{
Received 16th September 2008, Accepted 4th November 2008 \\ First published as an Advance Article on the web 25th November 2008 \\ DOI: $10.1039 / \mathrm{b816223a}$
}

\begin{abstract}
Microelectrode arrays find broad application in electroanalysis offering the enhanced sensitivity associated with microelectrodes, but with a high total current output. Such arrays are often constructed to make the electrodes 'diffusionally independent'. To emphasize that this is a time dependent property, a two-dimensional simulation, in conjunction with the diffusional domain approach, is used to model potential step transient currents at microelectrode arrays. Two types of array, hexagonal and cubic, are considered. In both cases the absolute (not relative) microelectrode separation distance has a significant effect on transient current. Three different regimes of transient current versus time can be observed at microelectrode arrays. At short times the transient response of isolated microelectrodes is seen, then at intermediate times the steady-state response of independent electrodes can be observed. At longer times planar diffusion to the entire array takes over. It follows that only at timescales corresponding to the first two regimes can the electrodes be considered as diffusionally independent. To verify the theory the potential step experiment is performed at a regularly spaced hexagonal iridium microdisk array. Theory is found to be in a good agreement with the experimental results.
\end{abstract}

\section{Introduction}

The development of lithography, nanoprinting, ${ }^{1}$ plasma etching, ${ }^{2}$ laser microstructuring ${ }^{3}$ and other techniques ${ }^{4-8}$ permits the fabrication of surfaces with a precisely defined geometry at the micro- or nano-scale that allows the fabrication of microelectrode arrays. Microelectrode arrays find wide application in electroanalysis due to their high current output as compared to a single microelectrode, whilst showing considerably less background/capacitative currents, as well as significantly enhanced sensitivity, in comparison to macroelectrodes. ${ }^{9-16}$ Considerable efforts have been devoted to designing new types of arrays containing micro-/nano-electrodes in both regular and random distributions. ${ }^{12,17-19}$ A critical factor in the design/fabrication of regular microdisk arrays is the center-to-center separation, $d$, between nearest neighbor electrodes. This needs to be large enough to avoid the overlap of adjacent diffusion zones but not too large as to result in inefficient use of space. ${ }^{20}$ Ideally the behavior of the microelectrode array should be similar to that of a single microelectrode, except in the magnitude of current. This requires that the diffusion layers of the microelectrodes in the array do not overlap. These conditions are referred to as diffusional independence. Such behavior can only be observed at timescales short compared to the time for molecule to diffuse the distance $d$. We note that earlier simulation work on potential

${ }^{a}$ Department of Chemistry, Physical and Theoretical Chemistry Laboratory, Oxford University, South Parks Road, Oxford, UK OX1 3QZ. E-mail: richard.compton@chem.ox.ac.uk; Fax: +44 (0) 1865 275410; Tel: +44 (0) 1865275413

${ }^{b}$ Centro National de Microelectronica, IMB-CNM. CSIC, Campus de la Universidad Auto'noma de Barcelona, Bellaterra 08193, Spain steps or cyclic voltammetry at arrays of microdisks has been reported. ${ }^{20-22}$

In this paper we use potential step chronoamperometry to investigate both theoretically and experimentally the concept of diffusional independence ${ }^{23,24}$ which has been well documented for isolated microelectrodes ${ }^{25-27}$ and also used to study microelectrode arrays. ${ }^{21,22}$ It is emphasized that the absolute distance $d$ controls the timescale of diffusional independence.

\section{Mathematical model and simulation procedure}

\subsection{Model of the electrode}

The idealized surface is composed of a regular array of microdisk electrodes (Fig. 1). We consider here two types of arrays: hexagonal and cubic. The diffusion of electrochemically active species to such a surface is complicated because it is intrinsically

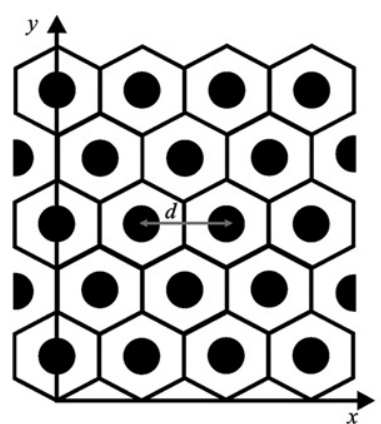

a)

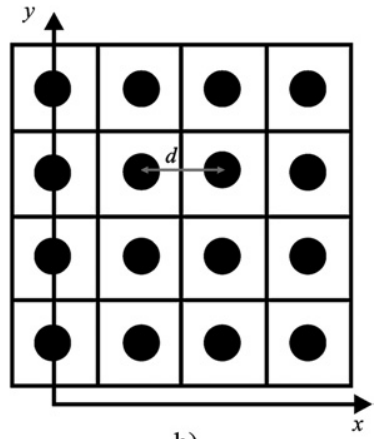

b)
Fig. 1 Conceptual rendering of hexagonal (a) and cubic (b) arrays of microdisk electrodes. 


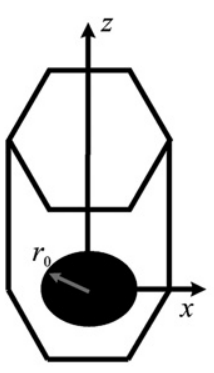

a)

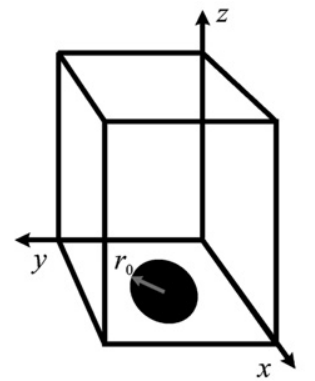

b)

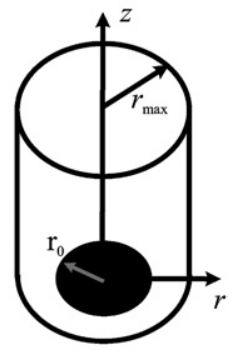

c)
Fig. 2 Unit cell in Cartesian coordinate (a) hexagonal array; (b) cubic array; (c) equivalent diffusion domain in cylindrical coordinates.

a three-dimensional problem. However, the problem can be simplified by noting that each electrode belongs to a diffusionally independent region known as a diffusion domain. ${ }^{28,29}$ The diffusion domain approximation treats these zones as being cylindrical with a microdisk center situated at the axis of symmetry, thus reducing the problem of diffusion to one of only two dimensions. The approximation is illustrated in Fig. 2. Fig. 2a and $2 b$ identify unit cells in Cartesian coordinates for hexagonal and cubic arrays. Fig. $2 \mathrm{c}$ shows the diffusional domain in cylindrical coordinates $(r, z)$. Note that the cylindrical coordinate system is defined relative to the single electrode domain. The cylindrical radial coordinate, $r$, is defined as the distance from the axis of symmetry that runs through the center of the electrode domain. The distance between microdisk centers in arrays is $d$. The area of cylindrical domain is chosen to be equal to the area of the unit cell in Cartesian coordinates:

$$
\frac{\sqrt{3}}{2} d^{2}=\pi r_{\max }^{2}
$$

for hexagonal and

$$
d^{2}=\pi r_{\max }^{2}
$$

for cubic arrays. The total current from the macroelectrode is given by simple multiplication of the current from a single diffusional domain to the total number of domain $N_{\mathrm{p}}$.

\subsection{Mathematical model}

Eqn (3) shows the electron transfer considered in these numerical simulations. Both species A and B are soluble, but only species $\mathrm{A}$ is assumed to be present in bulk solution.

$$
\mathrm{A}+\mathrm{e}^{-} \leftrightharpoons \mathrm{B}
$$

The mass transport of species A in cylindrical coordinates is described by Fick's second law of diffusion, which is presented in eqn (4). The computational model assumes the fully supported condition so there is no need to consider electromigration.

$$
\frac{\partial[\mathrm{A}]}{\partial t}=D_{\mathrm{A}}\left(\frac{\partial^{2}[\mathrm{~A}]}{\partial r^{2}}+\frac{1}{r} \frac{\partial[\mathrm{A}]}{\partial r}+\frac{\partial^{2}[\mathrm{~A}]}{\partial z^{2}}\right)
$$

where $D_{\mathrm{A}}$ is the diffusion coefficient of the species A and $t$ is time.

We assume that species $\mathrm{A}$ and $\mathrm{B}$ have equal diffusion coefficients:

$$
D_{\mathrm{A}}=D_{\mathrm{B}}=D
$$

so that at each point in solution the concentrations of species A and B satisfy the relationship:

$$
[\mathrm{A}]+[\mathrm{B}]=[\mathrm{A}]_{0}
$$

where $[\mathrm{A}]_{0}$ is the bulk concentration of $\mathrm{A}$. In such a situation the concentration profile of species A may be simulated independently from that of species B.

We next consider the potential step experiment applied to reduction of A. A typical chronoamperometric experiment involves a step from a potential of no current to one corresponding to a diffusion controlled rate. A potential step is therefore modeled simply by setting the concentration of $\mathrm{A}$, at the electrode surface equal to zero immediately after the step is implemented. This generates a singularity at $t=0$, where the flux is analytically infinite; in all cases, time steps used in the simulation were sufficiently dense to render any oscillations resulting from the $t=0$ singularity negligible in the region of interest.

The model is normalized using the dimensionless parameters in Table 1. The mass transport in cylindrical coordinates is described by the dimensionless variant of eqn (4):

$$
\frac{\partial a}{\partial \tau}=\frac{\partial^{2} a}{\partial R^{2}}+\frac{1}{R} \frac{\partial a}{\partial R}+\frac{\partial^{2} a}{\partial Z^{2}}
$$

\subsection{Boundary conditions and calculation of the flux normal to the electrode surface}

The boundary conditions for eqn (7) are summarized in Table 2.

\begin{tabular}{|c|c|}
\hline Boundary & Condition \\
\hline Initial concentrations & $a=1 ; b=0$ \\
\hline Axis of symmetry & $\frac{\partial a}{\partial R}=0 ; \quad \frac{\partial b}{\partial R}=0$ \\
\hline Diffusion domain borders & $\frac{\partial a}{\partial R}=0 ; \quad \frac{\partial b}{\partial R}=0$ \\
\hline Bulk solution concentration & $a=1 ; b=0$ \\
\hline Electrode surface concentration & $a=0$ \\
\hline
\end{tabular}
There is a no-flux condition at the axis of symmetry $(R=0)$ and at the diffusion domain border $R=R_{\max }$. The bulk solution condition is implemented at a distance $6 \sqrt{\tau_{\max }}$ from the electrode

Table 1 Dimensionless parameters used for numerical simulation

\begin{tabular}{ll}
\hline Parameter & Expression \\
\hline Radial coordinate & $R=r / r_{0}$ \\
Normal coordinate & $Z=z / r_{0}$ \\
Domain radius & $R_{\max }=r_{\max } / r_{0}$ \\
Time & $\tau=D t / r_{0}^{2}$ \\
Scan rate & $\sigma=F v r_{0}^{2} /(R T D)$ \\
Potential & $\theta=F\left(E-E_{\mathrm{f}}^{0}\right) /(R T)$ \\
Concentration of species A & $a=[\mathrm{A}] /[\mathrm{A}]_{0}$ \\
Concentration of species B & $b=[\mathrm{B}] /[\mathrm{A}]_{0}$ \\
Electrode flux & $j=-i /\left(F D[\mathrm{~A}]_{0} r_{0}\right)$ \\
\hline
\end{tabular}

Table 2 Boundary and initial conditions for eqn (7) 
surface, ${ }^{30}$ where $\tau_{\max }$ is the full time of the experiment. Beyond this the effect of diffusion is not important on the experimental timescale.

The electrode surface boundary conditions are given in Table 2. The dimensionless flux to a flat surface of cylindrical symmetry is given by eqn (8):

$$
j=2 \pi \int_{0}^{1} \frac{\partial a}{\partial Z} R \mathrm{~d} R
$$

\section{Experimental and computational details}

\subsection{Computation procedure}

Eqn (7) and its accompanying boundary conditions (Table 2) were discretised and solved by the alternating direction implicit (ADI) finite difference method ${ }^{31}$ in conjunction with the Thomas algorithm. ${ }^{32}$ The program was written in $\mathrm{C}++$. Because of the symmetry of the model, it is sufficient to solve the mass transport equations in the two-dimensional space in the region $0<R<$ $R_{\max }$ and $Z>0$. An expanding simulation grid is used in this work, which is based on the grids used in previous simulations of microdisk electrodes. ${ }^{33,34} \mathrm{~A}$ high mesh density is required where the electrode meets the symmetry axis and also close to the singularity where the electrode encounters the insulator.

The space grid used expands in both directions from $R=R_{0}$ and $Z=Z_{0}$ :

$$
\begin{aligned}
& R_{i+1}-R_{i}=h_{i} \\
& Z_{i+1}-Z_{i}=k_{i} \\
& h_{i}=\gamma_{R} h_{i-1} \\
& k_{i}=\gamma_{Z} k_{i-1}
\end{aligned}
$$

The standard values of the parameters used are $\gamma_{Z}=\gamma_{R}=1.125$ and $h_{0}=k_{0}=10^{-5}$.

An expanding time grid is also used; time steps were calculated each time in agreement with the expression:

$$
\min \left\{\tau_{k} \tau^{m}, \gamma_{\tau} \Delta \tau\right\}
$$

where, $\tau_{k}=0.01, m=0.5, \gamma_{\tau}=1.05$ and $\Delta \tau$ is the previous time step.

\subsection{Computational accuracy}

To check the convergence of the simulation procedure numerous simulations with different space grids and time steps were run. The calculated current converges with a decrease in the space and time steps, similar to the convergence presented in ref. 35 . To validate the procedure, comparisons to the known results for isolated single microdisk electrodes were made. The current in this case is given by the Shoup-Szabo equation; ${ }^{25,36}$ the comparison is presented in Fig. 3. It is seen that the results perfectly match each other. At short times the current decays in agreement with the dimensionless form of the Cottrell equation $^{36,37}(12)$ :

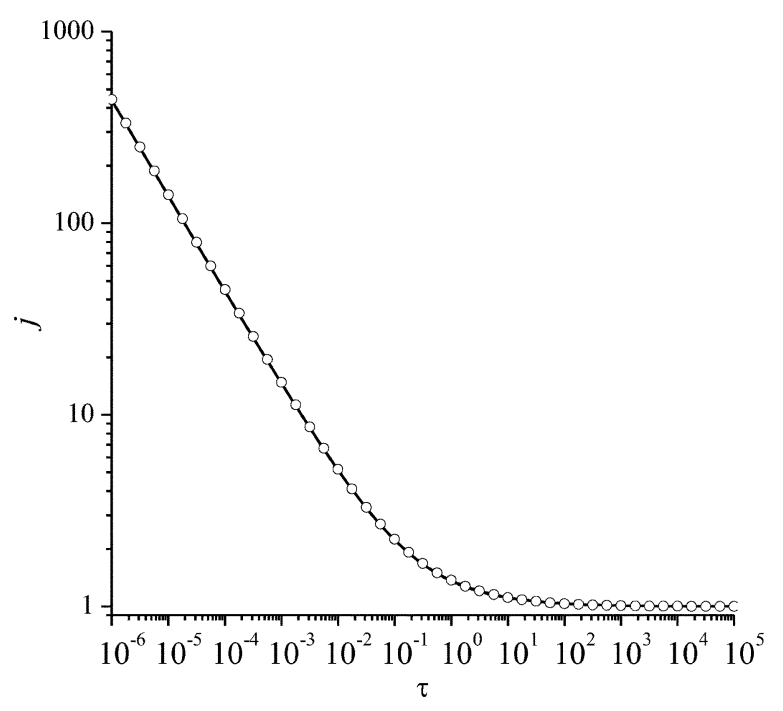

Fig. 3 Diffusional current versus dimensionless time in the potential step experiment. The solid line is our simulated transient for an isolated single microdisk electrode, the circles here were calculated with Shoup-Szabo equation..$^{25,36}$

$$
j=\frac{\pi^{1 / 2}}{4 \tau^{1 / 2}}
$$

\subsection{Chemical reagents and instrumentation}

All chemicals used were of analytical grade and were used as received without any further purification. These were: potassium chloride ( $\mathrm{KCl}$; Sigma-Aldrich), potassium ferrocyanide $\left(\mathrm{K}_{4} \mathrm{Fe}(\mathrm{CN})_{6}\right.$; $\mathrm{BDH}$ Chemicals Ltd, UK), copper sulfate $\left(\mathrm{CuSO}_{4}\right.$; Aldrich), sodium sulfate $\left(\mathrm{Na}_{2} \mathrm{SO}_{4} ;\right.$ Aldrich) and hydrochloric acid ( $\mathrm{HCl}$; Sigma-Aldrich). All solutions were prepared with deionized water of resistivity no less than $18.2 \mathrm{M} \Omega \mathrm{cm}$ at $25^{\circ} \mathrm{C}$ (Millipore water system, UK).

Voltammetric experiments were carried out with an $\mu$-Autolab II (Eco-Chemic, Utrecht, The Netherlands) connected to a PC using GPES (version 4.9) software for Windows. All measurements were conducted using a three electrode cell. Iridium microelectrode arrays were used as the working electrode. A saturated calomel electrode (SCE) was used as a reference electrode and a graphite rod as a counter electrode. The $\mathrm{pH}$ was monitored using a Hanna Instruments pH 213 Microprocessor $\mathrm{pH}$ Meter. The images of microdisk array surfaces were captured with a Sony XC-999P CCD camera attached to an optical OMV-

PAR microscope.

Prior to the voltammetric experiments, the system of $0.1 \mathrm{mM}$ $\mathrm{K}_{4} \mathrm{Fe}(\mathrm{CN})_{6} / 0.1 \mathrm{M} \mathrm{KCl}$ was degassed using purified $\mathrm{N}_{2}$. All experiments were conducted at $25 \pm 1{ }^{\circ} \mathrm{C}$.

Chronoamperometric transients for the oxidation of potassium ferrocyanide were recorded using different sample times: $0.05 \mathrm{~s}$ before the potential step and $0.0001,0.001,0.01$ or $0.05 \mathrm{~s}$ after the potential step. The potential was stepped from 0.1 to $0.3 \mathrm{~V}$.

In order to determine the true number of active microelectrodes on each array, copper metal was deposited onto the array surface via holding the potential at $-0.4 \mathrm{~V}$ for $20 \mathrm{~s}$ in a solution 


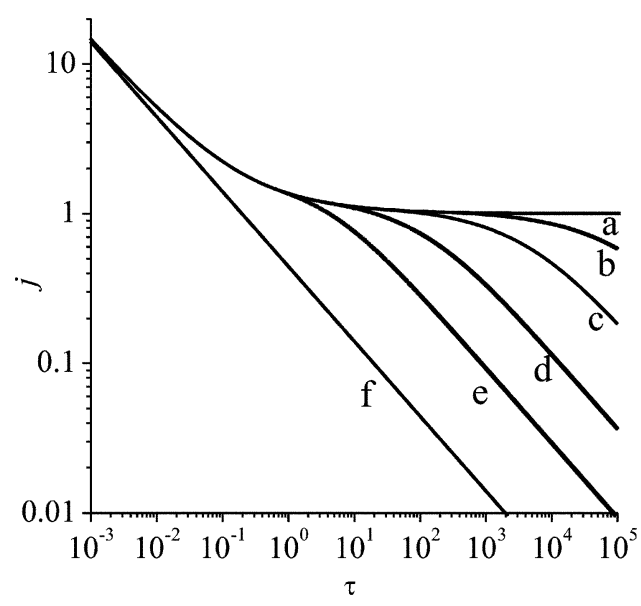

Fig. 4 Calculated dimensionless current versus dimensionless time in the potential step experiment: (a) non-interacting domains; (b) $R_{\max }=50$; (c) $R_{\max }=13$; (d) $R_{\max }=10$; (e) $R_{\max }=5$; (f) macroelectrode.

of $0.05 \mathrm{M} \mathrm{CuSO}_{4} / 0.1 \mathrm{M} \mathrm{Na}_{2} \mathrm{SO}_{4}(\mathrm{pH} \approx 3$, adjusted with $\mathrm{HCl})$. After copper deposition, the microelectrode surface was imaged with a camera attached to a microscope. This allowed us to undertake a visual count of the active microelectrodes on each array.

\subsection{Array fabrication}

The iridium microelectrode arrays $\left(r_{0}=2.5 \mu \mathrm{m}, d=55 \mu \mathrm{m}, N=\right.$ 2597) used in this study were made using standard photolithographic techniques. For the fabrication process Pyrex wafers

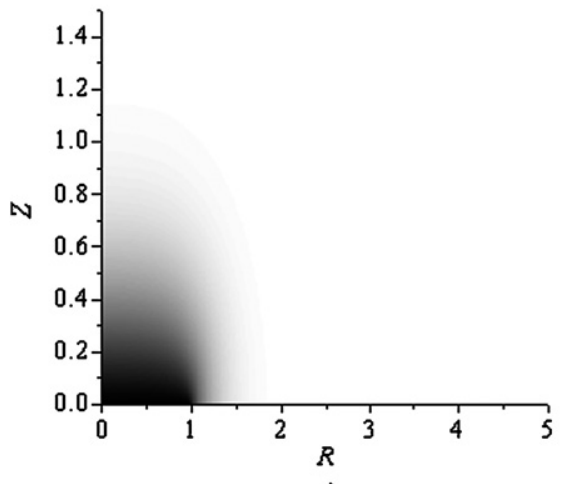

a)

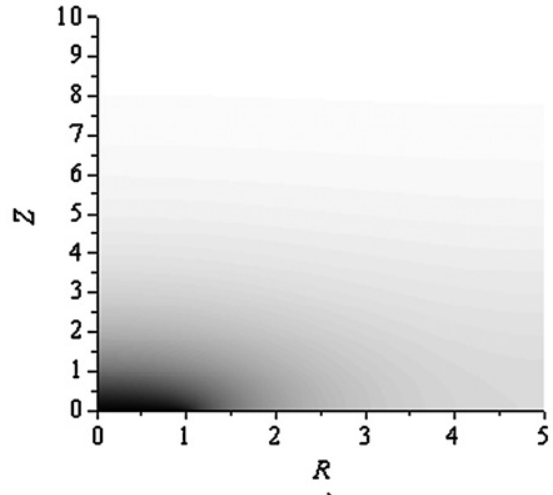

c) covered with a sacrificial layer of silicon oxide were used. Titanium $(20 \mathrm{~nm})$ and iridium $(100 \mathrm{~nm})$ layers were subsequently deposited and patterned by a 'lift-off' technique to form the electrodes and contact pads. The chips were cut individually from the wafer and mounted on test printed circuit boards. Following the soldering of wires to the connectors, a UV-curable polymer was used as encapsulant. The various processes employed are described in details elsewhere. ${ }^{23}$

\section{Results and discussion}

\subsection{The influence of $\boldsymbol{R}_{\max }$}

Simulated results for the potential step experiments are presented in Fig. 4. Values of the dimensionless current per single microdisk in an array are between the values of that from an isolated microdisk (curve (a) in Fig. 4) and that given by eqn (12), which corresponds to planar one-dimensional diffusion to a macroelectrode (curve (f) in Fig. 4). ${ }^{36,37}$

Considering Fig. 4, the higher the value of diffusion domain radius $R_{\max }$ the longer the time a microdisk in an array behaves as a diffusionally independent electrode. At long times, in all cases, deviation from the ideal microdisk behavior is observed due to overlapping of diffusional layers between microelectrodes in the array. Fig. 5 shows concentration profiles calculated for microdisk array with $R_{\max }=5$. At very small times $\tau<0.01$ the diffusion is planar and inefficient, and consequently a decrease of current is observed. ${ }^{36,37}$ At small times $\tau=0.1$ (Fig. 5a) and $\tau=1$ (Fig. 5b) the thickness of the diffusion layer is less than the separation between the electrodes in the array, and the current is

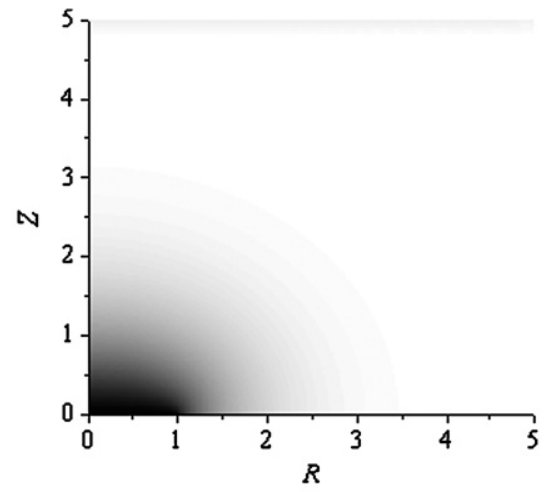

b)

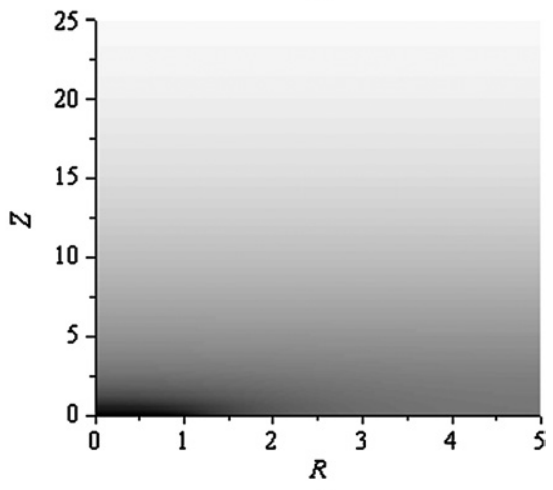

d)

Fig. 5 Simulated concentration profiles, $R_{\max }=5$ : (a) $\tau=0.1$; (b) $\tau=1$; (c) $\tau=10$; (d) $\tau=100$. The density of color identifies the concentration value. 
similar to that from a diffusionally independent microdisk (Fig. 4). On further increase of time the thickness of the diffusion layer gets larger and the diffusion layers of the microelectrodes in the array overlap (Fig. 5c); consequently the peak current deviates from the ideal behavior of a single isolated microdisk electrode. Further increase of the diffusion layer thickness with time causes a switch of diffusion type from twodimensional to effectively one-dimensional (Fig. 5d) and the current versus time in bi-logarithmic coordinates again has a slope of $-1 / 2$, corresponding to planar one-dimensional diffusion (eqn (12)).

\subsection{The influence of the microdisk radius and array symmetry}

We consider here two types of array symmetry: hexagonal and cubic (Fig. 1a and Fig. 1b). To characterize diffusional independence of microelectrodes in the array we introduce the time when the current reaches $90 \%$ of the steady-state current $t_{\mathrm{DI}}-$ time of diffusional independence:

$$
j\left(t_{\mathrm{DI}}\right) / N_{\mathrm{p}}=0.9 \times j_{\mathrm{ss}}
$$

where $j_{\mathrm{ss}}$ is the steady-state current at a single microdisk electrode, given by eqn $(14)^{36}$

$$
j_{\mathrm{ss}}=4 n F D C r_{0}
$$

where, $n$ is the number of electrons transferred, and $C$ is a bulk concentration of electroactive species.

In Table 3 the time of diffusional independence is presented for hexagonal and cubic arrays for different microelectrode separation distances $d$, and different microelectrode radii $r_{0}$. Table 3 shows that a decrease of radius causes a quadratic decrease in the time for diffusional independence; however, it also causes a quadratic decrease of the response time. ${ }^{40}$ Arrays with cubic symmetry have a slightly higher time of diffusional independence than hexagonal arrays with similar microdisk separation distance $d$, but the number of microdisks in the cubic array is smaller and consequently the total current is only $86 \%$ (eqn (15)) of that of a hexagonal array of the same area:

$$
N_{\mathrm{pc}}=\frac{\sqrt{3}}{2} N_{\mathrm{ph}}=0.86 \times N_{\mathrm{ph}}
$$

where, $N_{\mathrm{pc}}$ and $N_{\mathrm{ph}}$ are the number of microelectrodes in the

\begin{tabular}{|c|c|c|c|c|c|c|}
\hline \multirow[b]{2}{*}{$d / r_{0}{ }^{a}$} & \multicolumn{3}{|c|}{ Hexagonal } & \multicolumn{3}{|l|}{ Cubic } \\
\hline & $\begin{array}{l}r_{0}= \\
100 \mathrm{~nm}\end{array}$ & $\begin{array}{l}r_{0}= \\
1 \mu \mathrm{m}\end{array}$ & $\begin{array}{l}r_{0}= \\
10 \mu \mathrm{m}\end{array}$ & $\begin{array}{l}r_{0}= \\
100 \mathrm{~nm}\end{array}$ & $\begin{array}{l}r_{0}= \\
1 \mu \mathrm{m}\end{array}$ & $\begin{array}{l}r_{0}= \\
10 \mu \mathrm{m}\end{array}$ \\
\hline 5 & $6 \times 10^{-5}$ & 0.006 & 0.6 & $7.2 \times 10^{-5}$ & 0.0072 & 0.72 \\
\hline 10 & $6 \times 10^{-4}$ & 0.04 & 4 & $4.9 \times 10^{-4}$ & 0.049 & 4.9 \\
\hline 50 & 0.055 & 5.5 & 550 & 0.072 & 7.2 & 720 \\
\hline
\end{tabular}
cubic and hexagonal arrays respectively.

Table 3 Timescale of diffusional independence

${ }^{a} R_{\max }$ values for hexagonal and cubic arrays can be calculated from these data according eqns (1) and (2) respectively.

\subsection{Experimental validation}

Fig. 6 shows the cyclic voltammograms recorded at $100 \mathrm{mV} \mathrm{s}^{-1}$ in $1.0 \mathrm{mM}$ ferrocyanide/ $0.1 \mathrm{M} \mathrm{KCl}$ at a hexagonal iridium microelectrode array $\left(r_{0}=2.5 \mu \mathrm{m}, d=55 \mu \mathrm{m}, N=2597\right)$. The steadystate current to the iridium array calculated as the sum of the steady-state currents to 2597 diffusionally independent microelectrodes is $1.6 \mu \mathrm{A}$, so it is obvious that only some of the microelectrodes in the array contribute to the current. It is known that lithographic procedures commonly lead to a significant number of 'dead' electrodes. ${ }^{39}$

To model the transient, the diffusion coefficient of ferrocyanide was taken as $6.5 \times 10^{-6} \mathrm{~cm}^{2} \mathrm{~s}^{-1} \cdot{ }^{38} R_{\max }$ and $N$ were varied to produce the best fit, including under steady-state 'diffusionally independent' conditions subjected to the condition of eqn (16). We assume that the presence of electroinactive disks does not significantly affect the symmetry.

$$
\pi N R_{\max }^{2}=2597 \times \frac{\sqrt{3}}{2} \times d^{2}
$$

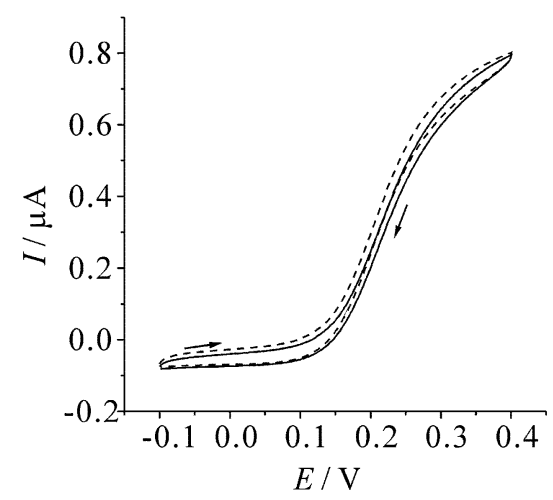

Fig. 6 Cyclic voltammogram of $1.0 \mathrm{mM}$ ferrocyanide in $0.1 \mathrm{M} \mathrm{KCl}$ obtained at an iridium microdisk electrode array. The scan rate is $100 \mathrm{mV}$ $\mathrm{s}^{-1}$. The dashed and solid curves represent respectively cyclic voltammograms obtained before and after chronoamperometric measurements.
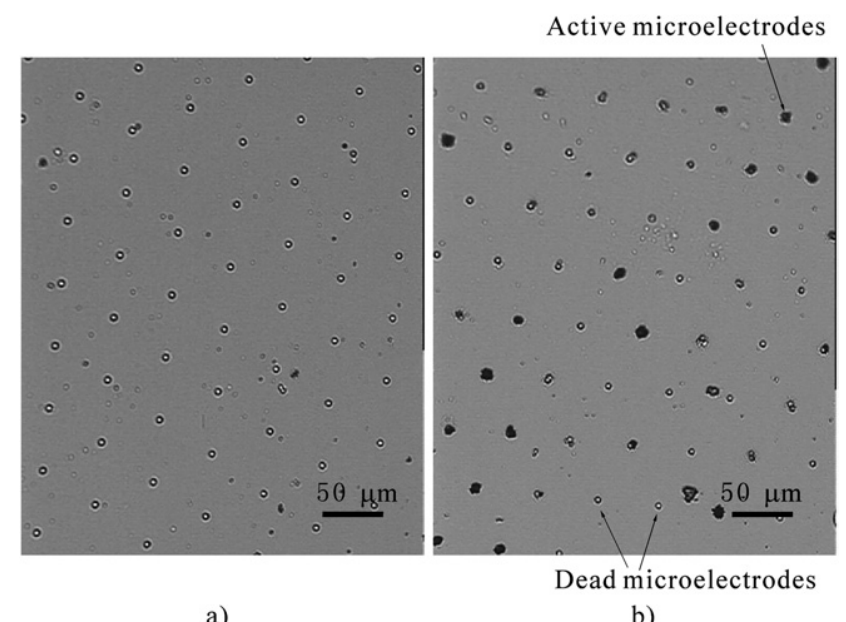

Fig. 7 Microscopy image of detailed area of iridium microelectrode arrays: (a) before copper deposition; (b) after $20 \mathrm{~s}$ of copper deposition at $-0.4 \mathrm{~V} v s$. SCE. 


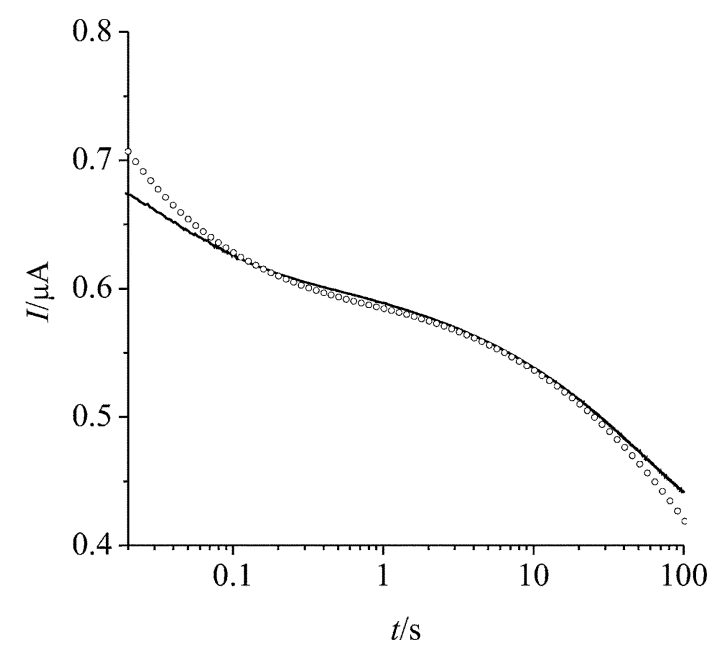

Fig. 8 Total current from iridium microelectrode array registered experimentally $(-)$ in a potential step experiment, where the potential is stepped from 0.1 to $+0.3 \mathrm{~V}$. Circles $(\mathrm{O})$ represent the best theoretical fit.

The optimized values were $N=900$ and $R_{\max }=20$. The value of $N$ suggest that $c a .35 \%$ of the disks are electroactive and that the remainder are 'dead'. To independently verify the value the disks were electroplated with copper, so that copper was only deposited on the active disks. ${ }^{39}$ Fig. 7 shows a fragment of the microdisk electrode array before and after copper deposition. Active electrodes can be visually distinguished from 'dead'. Microscopic investigation of the whole surface showed that $c a$. $31 \%$ of the disks are active, validating the analysis presented above.

Returning to Fig. 8 we see deviation between theory and experiment both at very short and very long times. The latter is likely due to the increased mass transport efficiency by natural convection for times of $\sim 10-100 \mathrm{~s},{ }^{36}$ whilst the former is attributed either to kinetic limitation and/or ohmic losses over the electrode surface.

\section{Conclusions}

Three different time regimes are observed in a potential step experiment at a microelectrode array. First a rapid $\left(j \propto \tau^{-1 / 2}\right)$ decrease of current is seen at short times as is also observed for isolated microdisks. Second a steady-state current is observed and finally a decrease of current is seen at long times. The length of the steady-state period is strongly dependent on the diffusional domain size $R_{\max }$ and for $R_{\max }<5$ a steady-state regime is not observed. All three regions of behavior were observed experimentally on an iridium microelectrode array. Table 3 reports the approximate timescales for diffusional independence. Note that even for electrodes as large as $1 \mu \mathrm{m}$ the timescale of diffusional independence is less than $10 \mathrm{~s}$ unless unusually large separation distances $(\gg 100 \mu \mathrm{m})$ are used. Finally we emphasize that the concept of 'diffusional independence' can only apply to an electrode array for a finite timescale.

\section{Acknowledgements}

D. M. thanks St. John's College, Oxford, for a Kendrew Scholarship. N. V. R. and X.-J. H. thank EPSRC for support.

\section{References}

1 M. Ben Ali, T. Ondarcuhu, M. Brust and C. Joachim, Langmuir, 2002, 18, 872 .

2 H. Masuda, M. Watanabe, K. Yasui, D. Tryk, T. Rao and A. Fujishima, Adv. Mater., 2000, 12, 444.

3 B. K. Nayak, M. C. Gupta and K. W. Kolasinski, Nanotechnology, 2007, 18, 195302.

4 A. N. Shipway, E. Katz and I. Willner, ChemPhysChem, 2000, 1, 18.

5 A. Haryono and W. H. Binder, Small, 2006, 2, 600.

6 S. Il Yoo, J. H. Kwon and B. H. Sohn, J. Mater. Chem., 2007, 17, 2969.

7 F. Javier del Campo, J. García-Céspedes, F. J. Muñoz and E. Bertran, Electrochem. Commun., 2008, 10, 1242.

8 O. Orgeig, N. Godino, F. Javier del Campo, F. J. Muñoz, F. Nikolajeff and L. Nyholm, Anal. Chem., 2008, 80, 3622.

9 A. Stett, U. Egert, E. Guenther, F. Hofmann, T. Meyer, W. Nisch and H. Haemmerle, Anal. Bioanal. Chem., 2003, 377, 486.

10 A. O. Simm, C. E. Banks, S. Ward-Jones, T. J. Davies, N. S. Lawrence, T. G. J. Jones, L. Jiang and R. G. Compton, Analyst, 2005, 130, 1303.

11 M. E. Sandison, N. Anicet, A. Glidle and J. M. Cooper, Anal. Chem., $2002,74,5717$.

12 S. Fletcher and M. D. Horne, Electrochem. Commun., 1999, 1, 502.

13 O. Ordeig, J. del Campo, F. X. Muñoz, C. E. Banks and R. G. Compton, Electroanalysis, 2007, 19, 1973.

14 A. Berduque, Y. H. Lanyon, V. Beni, G. Herzog, Y. E. Watson, K. Rodgers, F. Stam, J. Alderman and D. W. M. Arrigan, Talanta, 2007, 71, 1022.

15 D. W. M. Arrigan, Analyst, 2004, 129, 1157.

16 V. Beni and D. W. M. Arrigan, Curr. Anal. Chem., 2008, 4, 229.

17 M. Pagels, C. E. Hall, N. S. Lawrence, A. Meredith, T. G. J. Jones, H. P. Godfried, C. S. J. Pickles, J. Wilman, C. E. Banks, R. G. Compton and L. Jiang, Anal. Chem., 2005, 77, 3705.

18 C. A. Huber, T. E. Huber, M. Sadoqi, J. A. Lubin, S. Manalis and C. B. Prater, Science, 1994, 263, 800.

19 B. J. Seddon, Y. Shao and H. H. Girault, Electrochim. Acta, 1994, 39, 2377.

20 T. J. Davies and R. G. Compton, J. Electroanal. Chem., 2005, 585, 63.

21 C. Beriet, R. Ferrigno and H. H. Girault, J. Electroanal. Chem., 2000, 486, 56.

22 H. J. Lee, C. Beriet, R. Ferrigno and H. H. Girault, J. Electroanal. Chem., 2001, 502, 138.

23 T. J. Davies, S. Ward-Jones, C. E. Banks, J. del Campo, R. Mas, F. X. Muñoz and R. G. Compton, J. Electroanal. Chem., 2005, 585, 51.

24 I. Streeter, N. Fietkau, J. Del Campo, R. Mas, F. X. Muñoz and R. G. Compton, J. Phys. Chem. C, 2007, 111, 12058.

25 D. Shoup and A. Szabo, J. Electroanal. Chem., 1982, 140, 237.

26 C. Amatore and I. Svir, J. Electroanal. Chem., 2003, 557, 75.

27 O. V. Klymenko, R. G. Evans, C. Hardacre, I. B. Svir and R. G. Compton, J. Electroanal. Chem., 2004, 571, 211.

28 B. A. Brookes, T. J. Davies, A. C. Fisher, R. G. Evans, S. J. Wilkins, K. Yunus, J. D. Wadhawan and R. G. Compton, J. Phys. Chem. B, 2003, 107, 1616.

29 C. Amatore, J. M. Saveant and D. Tessier, J. Electroanal. Chem., 1983, 147, 39.

30 I. B. Svir, O. V. Klymenko and R. G. Compton, Radiotekhnika, 2001, 118, 92.

31 J. Peaceman and H. Rachford, J. Soc. Ind. Appl. Math., 1955, 3, 28.

32 K. E. Atkinson, Elementary Numerical Analysis, John Wiley and Sons, New York 2004.

33 D. J. Gavaghan, J. Electroanal. Chem., 1998, 456, 1.

34 D. J. Gavaghan, J. Electroanal. Chem., 1998, 456, 13.

35 D. Menshykau, I. Streeter and Richard G. Compton, J. Phys. Chem. C, 2008, 112, 14428.

36 A. J. Bard and L. R. Faulkner, Electrochemical Methods: Fundamentals and Applications, Wiley \& Sons, Inc, 2nd edn, 2001.

37 F. G. Cottrell, Z. Physik. Chem., 1902, 42, 385.

38 M. von Stackelberg, M. Pilgram and W. Toome, Electrochemistry, 1953, 57, 342.

39 O. Ordeig, C. E. Banks, T. J. Davies, J. del Campo, R. Mas, F. X. Munoz and R. G. Compton, Analyst, 2006, 131, 440.

40 R. G. Compton and C. E. Banks, Understanding Voltammetry, World Sientific, Singapore, 2007. 